\title{
Catheter ablation for successful management of left posterior fascicular tachycardia: an approach guided by recording of fascicular potentials
}

Demosthenes Katritsis, Spencer Heald, Arif Ahsan, Mark H Anderson, A John Camm, David E Ward, Edward Rowland

\begin{abstract}
Objective-To assess whether catheter ablation of fascicular tachycardia can be facilitated by the recording of sharp deflections arising from the mid-septum-inferior apical septum of the left ventricle.

Patients and methods-Seven consecutive patients (mean age 29 (range 16-43) years) with ventricular tachycardia originating from the left posterior fascicle underwent electrophysiology study and detailed mapping of endocardial activation. Selection of ablation sites in the last five patients was based on the recording, during left posterior fascicular tachycardia and sinus rhythm, of a discrete potential preceding the earliest ventricular electrogram, which was thought to represent conduction through the posterior fascicle.

Results-Patients were treated with low energy direct current or radiofrequency current ablation. The median fluoroscopy and procedure times were 23 (range 6-42) min and 110 (range 50-176) $\mathrm{min}$, respectively. In a follow up period of 4 to 16 months, six patients were asymptomatic and one had minor symptoms. No patient had any change in intraventricular conduction. Similar potentials were also recorded from the left posterobasal septum in three of eight patients who underwent catheter ablation of left free wall accessory pathways.

Conclusion-Fascicular potentials can be reproducibly recorded in left posterior fascicular tachycardia and may serve as a reliable marker for successful ablation procedures. The relation of these potentials with the substrate of the tachycardia, however, remains obscure.
\end{abstract}

(Heart 1996;75:384-388)

Cardiological

Sciences, St George's

Hospital Medical

School, London

D Katritsis

$S$ Heald

A Ahsan

M H Anderson

A J Camm

D E Ward

E Rowland

Correspondence to:

Dr D Ratritsis, Onassis Cardiac Surgery Center,

356 Sygrou Ave,

Athens 176 74, Greece.

Accepted for publication 9 October 1995

Keywords: catheter ablation; fascicular tachycardia; fascicular potentials

Fascicular tachycardias are "idiopathic", sustained tachycardias which probably originate from microre-entry within one of the fascicles of the left bundle branch. ${ }^{1-3}$ Occasional attempts at direct current (DC) energy or radiofrequency catheter ablation of the origin of fascicular tachycardia have been reported previously ${ }^{4-8}$ and recently, we and others suggested that the successful ablation site is indi- cated by the recording of sharp deflections arising from the midseptum-inferior apical septum of the left ventricle..$^{9-11}$ Although these potentials seem to originate from the Purkinje network of the left posterior fascicle, their functional significance as well as their relation with the substrate of the tachycardia itself remains obscure. ${ }^{1011}$

We report on our experience from the successful application of low energy DC and radiofrequency current ablation for the treatment of seven consecutive patients with posterior fascicular tachycardia which, although responsive to intravenous verapamil, was not controlled by oral treatment. In the last five patients the selection of ablation sites was guided by the recording of a discrete potential preceding the earliest ventricular electrogram and corresponding anatomically to the location of the posterior fascicle of the left bundle branch.

\section{Patients and methods}

PATIENTS

From September 1990 to April 1994, seven patients (four men and three women with a mean age of 29 years) with recurrent, sustained ventricular tachycardia and otherwise normal hearts were treated with catheter ablation at St George's and Royal Brompton Hospitals, London. Patients presented with sustained ventricular tachycardia with a 12 lead electrocardiogram (ECG) morphology of right bundle branch block (RBBB) and left axis deviation (table and fig 1). All patients had unremarkable physical examination and a normal resting ECG, chest radiograph, echocardiogram, and exercise tests. Coronary angiography performed in one patient (no 1) was also normal. All patients had been highly symptomatic with frequent palpitations and presyncopal attacks. Oral treatment with various antiarrhythmic drugs, including verapamil, sotalol, flecainide, and disopyramide and, in one patient amiodarone, was ineffective or could not be tolerated at maximum doses.
ELECTROPHYSIOLOGICAL STUDY AND CATHETER ABLATION

General (for low energy shocks) or local (for radiofrequency current) anaesthesia was administered, and under fluoroscopic control a Josephson quadripolar catheter was inserted for high right atrial or right ventricular pacing, a tripolar or hexapolar catheter was positioned in the His area or the coronary sinus, or both, 
Electrophysiological data

\begin{tabular}{|c|c|c|c|c|c|c|c|c|c|c|c|}
\hline Patient no & $\begin{array}{l}\text { Age Sex } \\
\text { (years) }\end{array}$ & $\begin{array}{l}\text { Mode of } V T \\
\text { induction }\end{array}$ & $\begin{array}{l}V T \text { cycle } \\
\text { length }\end{array}$ & $E C G$ & $\begin{array}{l}\text { HV interval } \\
(m s)\end{array}$ & $\begin{array}{l}Q R S \\
(m s)\end{array}$ & Energy & $\begin{array}{l}\text { Selection of ablation } \\
\text { sites }\end{array}$ & $\begin{array}{l}\text { FV interval } \\
(m s)\end{array}$ & $\begin{array}{l}\text { Follow up } \\
\text { (months) }\end{array}$ & Outcome \\
\hline 1 & $37 \mathrm{~F}$ & $\mathrm{RAP}+$ isopr & 230 & $\mathrm{RBBB}+\mathrm{LAD}$ & Negative & 130 & LE & $\begin{array}{l}\text { Earliest activation/ } \\
\text { pace map }\end{array}$ & $20^{\star}$ & 16 & Asymptomatic \\
\hline 2 & $16 \mathrm{~F}$ & RAP & 260 & $\mathrm{RBBB}+\mathrm{LAD}$ & -20 & 120 & LE/RF & $\begin{array}{l}\text { Earliest activation/ } \\
\text { pace map }\end{array}$ & $25^{\star}$ & 15 & $\begin{array}{l}\text { Well receiving } \\
\text { disopyramide }\end{array}$ \\
\hline 3 & $23 \mathrm{M}$ & V stim & 340 & RBBB+LAD & -20 & 130 & LE & Fascicular potential & 25 & 16 & Asymptomatic \\
\hline 4 & $19 M$ & V stim & 240 & $\mathrm{RBBB}+\mathrm{LAD}$ & Negative & 130 & LE & Fascicular potential & 23 & 10 & Asymptomatic \\
\hline 5 & $39 M$ & V stim & 320 & RBBB+LAD & -35 & 120 & LE & Fascicular potential & 25 & 12 & Asymptomatic \\
\hline 6 & $25 \mathrm{~F}$ & V stim & 440 & $\mathrm{RBBB}+\mathrm{LAD}$ & -20 & 130 & RF & Fascicular potential & 38 & 4 & Asymptomatic \\
\hline 7 & $43 \mathrm{M}$ & V stim & 320 & RBBB+LAD & Negative & 120 & RF & Fascicular potential & 35 & 6 & Asymptomatic \\
\hline
\end{tabular}

RAP, Rapid atrial pacing; isopr, isoprenaline infusion; LAD, left axis deviation; VT, ventricular tachycardia; $V$ stim, programmed ventricular stimulation; LE, low energy; RF, radiofrequency; FV, fascicular potential to surface QRS. ^Ventricular electrogram to surface QRS.

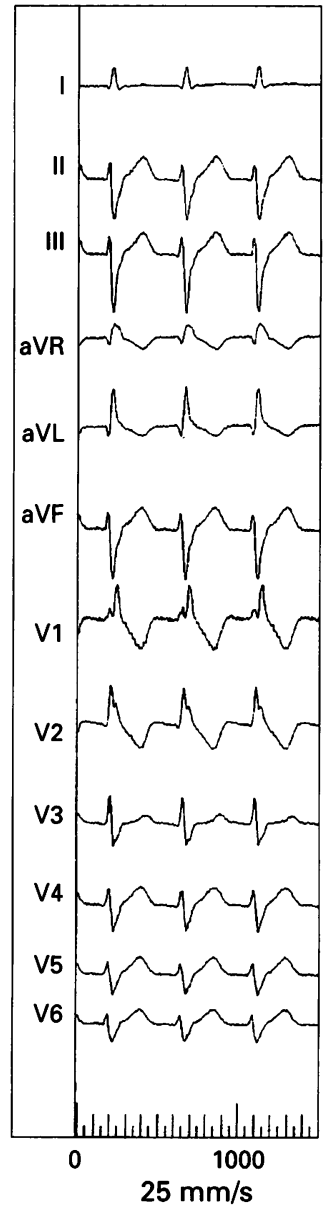

Figure 1 Twelve lead ECG recorded during fascicular tachycardia. and the mapping/ablating catheter was positioned in the inferobasal area of the left side of the septum. For the first three patients bipolar electrograms were recorded from the distal pair of electrodes, filtered at $30-500 \mathrm{~Hz}$, amplified at gains of $20-80 \mathrm{~mm} / \mathrm{mV}$, and printed at a paper speed of $100 \mathrm{~mm} / \mathrm{s}$ using a multichannel-ink jet recorder (Mingograf 7, Siemens-Elema). For the last four patients a Bard electrophysiology computer-assisted system was used and continuous recording of electrograms and surface ECG was stored on a hard disk.

A National Heart Hospital bipolar catheter (patient nos 1 and 2) or a deflectable catheter with a $4 \mathrm{~mm}$ tip and $2.5 \mathrm{~mm}$ interelectrode spacing (Webster or Medtronic RF Ablater) was used for mapping and ablation. Low energy was delivered by the special National Heart Hospital low energy ablator in the form of multiple low energy shocks. ${ }^{10}$ Radiofrequency current was supplied by a 500 $\mathrm{kHz}$ generator (HAT $200 \mathrm{~S}$, Dr Osypka $\mathrm{GmbH}$ or Medtronic Atakr) at constant preset electrical power usually ranging from 30 to 50 W. Current impedance or catheter tip temperature was monitored throughout the current discharges.

\section{Results}

ELECTROPHYSIOLOGICAL DATA

The table indicates the electrophysiological data of the patients. All patients had decremental antegrade and retrograde conduction curves with normal HV intervals during sinus rhythm. Tachycardia could be induced by atrial extrastimulation in two patients. In the other five tachycardia was inducible by ventricular extrastimulation but in three of them it could be terminated by rapid atrial pacing. There were periods of atrioventricular (AV) dissociation in all patients during tachycardia and in two of them atria extrastimuli could dissociate the His bundle electrogram without resetting the tachycardia. There was no consistent relation between the initiating interval and the first tachycardia cycle length. The His bundle electrogram could be recorded in four patients during tachycardia, being obscured by the ventricular electrogram in the remaining three. The HV interval, therefore, was presumed to be negative in all patients. Intravenous verapamil given during tachycardia was successful in restoring sinus rhythm in all patients, although not invariably in patient no 3.

\section{SELECTION OF ABLATION SITES}

Tachycardia was induced after the position of the ablating catheter in the left posterobasal/apical septum had been verified using the right anterior oblique, left anterior oblique, and anteroposterior radiographic views (fig 2). Mapping was started and potential ablation sites were sought by detecting the point of earliest activation of the ventricular electrogram as compared with the QRS complex of the surface ECG (table). In the last five patients, a discrete potential of between 300 and $400 \mu \mathrm{V}$ was reproducibly recorded immediately before the earliest ventricular activation (fig 3) during tachycardia. During sinus rhythm this potential was also recorded at the same ventricular site and could be recorded slightly earlier or later by marginally withdrawing or advancing the catheter towards the apex, respectively. Ventricular pacing during sinus rhythm at a rate similar to that during tachycardia confirmed concordance between paced and tachycardia ECGs. No fragmentation or delayed potentials could be recorded by the mapping/ablation electrode at the same ventricular sites during sinus rhythm. In patient no 1 the tachycardia was not easily inducible, and, most of the time, it was nonsustained, thus prohibiting detailed mapping. Ablation sites, therefore, were mainly chosen according to pace mapping results. Three attempts were undertaken in patient no 2 , one with radiofrequency and two with low energy.

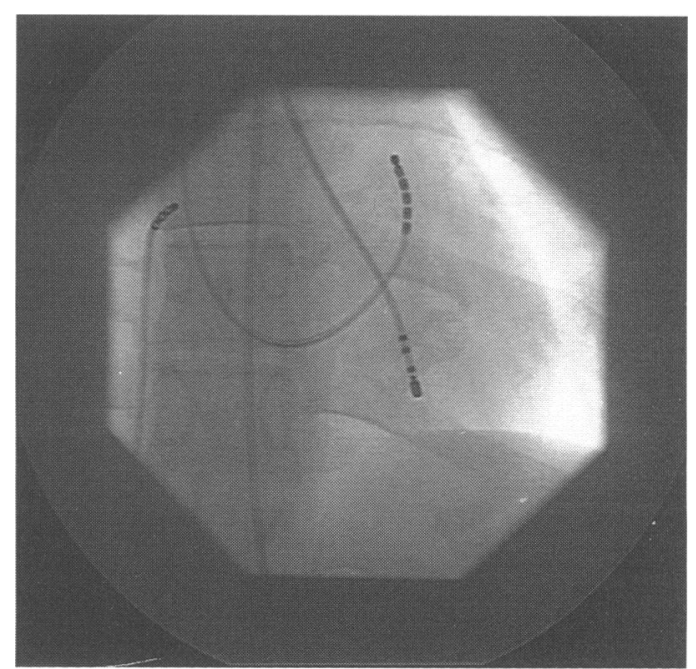

Figure $2 P A$ view of the position of the catheters during ablation. A fosephson quadripolar electrode is positioned in the right atrium, a hexapolar electrode in the coronary sinus, and the ablating catheter in the left ventricular septum. 
A

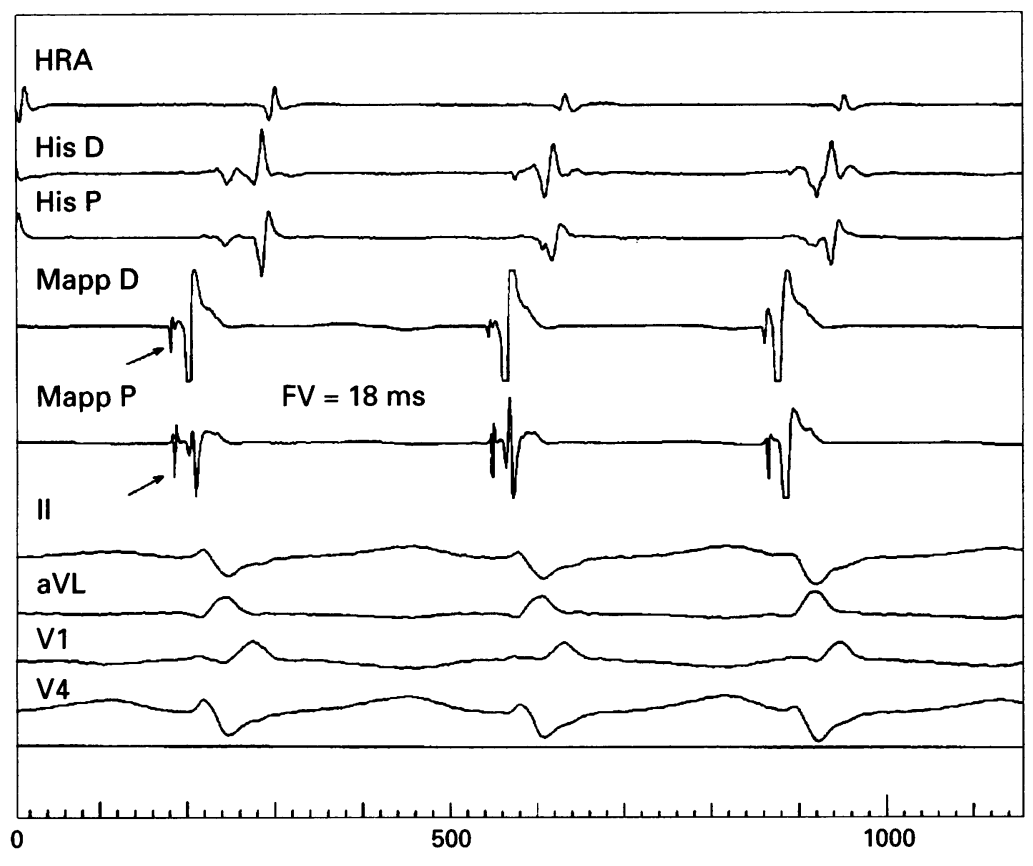

B

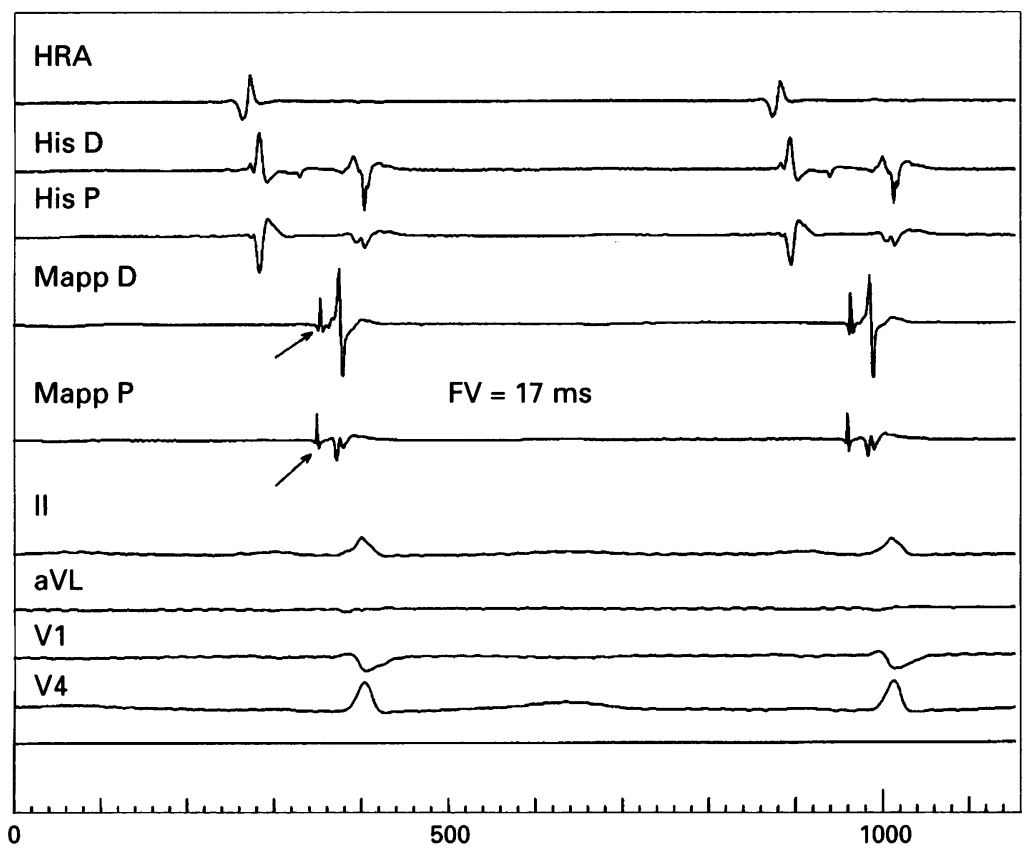

Figure 3 Mapping during $(A)$ fascicular tachycardia and $(B)$ sinus rhythm. A discrete potential (fascicular potential?) preceding the earliest ventricular electrogram is indicated.

Ablation sites were selected by earliest activation and pace mapping in all patients. The stimulus to QRS delay in all patients ranged between 20 and $30 \mathrm{~ms}$. Two attempts were also required in patient no 5 . During the first ablation, a fascicular potential was recorded $18 \mathrm{~ms}$ in front of the surface QRS during tachycardia and although ablation was initially successful, the arrhythmia recurred in the form of non-sustained runs and frequent ventricular extrasystoles of the same morphology (RBBB and left axis deviation). At the second attempt mapping was based on ventricular extrasystoles and the recorded potential preceded the surface QRS by $35 \mathrm{~ms}$.

Radiofrequency was the only form of energy for ablation in patient nos 6 and 7, while in the remaining patients, 8-10 low energy shocks of 5-25 J were delivered. In all patients, $30 \mathrm{~min}$ after the last energy discharge, tachycardia could not be induced with and without isoprenaline infusion $(2 \mu \mathrm{g} / \mathrm{min})$ and by either atrial or ventricular stimulation. The median fluoroscopy time was 23 (range $6-42 \mathrm{~min}$ ) and the median procedure time was 110 (range 50-176) min. The next day the stimulation test was repeated and confirmed noninducibility of the tachycardia. No axis deviation was documented on the 12 lead ECG.

FASCICULAR POTENTIALS IN PATIENTS

WITHOUT FASCICULAR TACHYCARDIA

Limited mapping was also undertaken in eight patients who underwent catheter ablation of left free wall accessory pathways. Fascicular potentials could be reproducibly recorded in three of them; in all three patients stimulation of the ventricle at that site produced extrasystoles with typical "RBBB with left axis" morphology.

\section{FOLLOW UP}

Patients have been followed up for a period of 4-16 months (table). Patient no 2 had two previous attempts which kept her asymptomatic for about seven months and one year respectively. Since the last attempt she has occasionally experienced short lived episodes of palpitations which, however, are less disturbing than previously and are well controlled with oral disopyramide. The other patients have been asymptomatic.

\section{Discussion}

FASCICULAR TACHYCARDIA

Several reports have now established fascicular tachycardia as a recognised clinical entity. The posterior fascicle is usually involved resulting in a tachycardia with RBBB and left axis deviation but patients with inferior axis ${ }^{3}$ - that is, probably of anterior fascicular origin, or with intermediate axis ${ }^{11}$ have also been described. Idiopathic left ventricular tachycardia in general, can also originate from the left free ventricular wall ${ }^{11}$ producing a rightward axis. Re-entry rather than automaticity within one of the left bundle fascicles ${ }^{23}$ has been proposed as the most plausible mechanism. However, the arrhythmia responds to verapamil ${ }^{2812}$ and the mechanism is obscure. The arrhythmia usually occurs in young patients with otherwise normal hearts and who can tolerate the tachycardia rates usually ranging from 140 to 220 beats/min.

\section{SELECTION OF ABLATION SITES}

In large re-entry circuits such as those arising from infarct scars, areas of slow conduction during sinus rhythm can be identified by eliciting entrainment or concealed entrainment, ${ }^{13}$ and detecting fractionated electrograms ${ }^{14}$ or mid-diastolic potentials. ${ }^{15}$ Ablation at these sites has resulted in a significantly lower recurrence rate than ablations performed at areas where the earliest endocardial activation was 
recorded. ${ }^{16}$ In accordance with other reports ${ }^{3711}$ we failed to record any fragmentation which should indicate the area of slow conduction either during sinus rhythm or tachycardia. In contrast to patients with coronary artery disease, patients with structurally normal hearts and ventricular tachycardia may have a relatively small area of slow conduction which produces enough delay for re-entry without displaying prominent and easily recordable fragmentation. ${ }^{3}$ Alternatively, the regions of slow conduction and endocardial exit may be close or identical. ${ }^{17}$

Recording of the earliest activation during tachycardia in the posterobasal septum and pace mapping have been used to select ablation target sites in fascicular tachycardia. ${ }^{48-11}$ As also occurs in right ventricular outflow tract tachycardia, ${ }^{17} 18$ the earliest endocardial activation time (about 20-25 ms before the onset of the earliest QRS in our series) is considerably later than in patients with sustained ventricular tachycardia caused by ischaemic heart disease (50-100 ms). Optimal pace mapping also has a short stimulus to QRS interval (20-30 $\mathrm{ms}$ in our series) in fascicular as well as in right ventricular outflow tract tachycardia. ${ }^{18}$ Nakagawa et al ${ }^{10}$ achieved ECG concordance during pace mapping also at sites adjacent to the site of successful ablation, thus suggesting that pace mapping may have limited value in this arrhythmia. However, pace mapping has been successfully employed by other groups 1119 and, in particular, Wen et al ${ }^{11}$ achieved successful ablation even at sites where the pace map ECG, although not similar, closely resembled the ECG during tachycardia. In our second patient, ablation attempts based only on earliest activation or pace mapping without seeking fascicular potentials were not successful in abolishing symptoms.

\section{RECORDING OF FASCICULAR POTENTIALS}

Our series confirms that recording of potentials representing the activation of fascicular tissue is feasible in patients with fascicular tachycardia. In one of the two reported cases by Page et $a l^{7}$ a similar potential was also clearly recorded during tachycardia at the site of successful energy delivery. Previous multiple radiofrequency energy discharges were unsuccessful in interrupting tachycardia despite early local electrical activity (preceding the QRS by $30-40 \mathrm{~ms}$ ) and pace mapping concordance. Although these potentials are thought to originate from the Purkinje network of the left posterior fascicle, ${ }^{10} 11$ their exact nature as well as their relation with the substrate of the tachycardia itself remain obscure. It is not known whether such potentials represent specialised conduction tissue close to or part of the His-Purkinje system. Further studies are required to distinguish whether this is a normal part of the left fascicle or represents a left sided fasciculoventricular bypass tract. The fact that we did not detect axis deviation in any of our patients after the procedure could be compatible with either ablation of accessory tissue or modification of the re-entrant circuit. In the rare reported cases of idiopathic left ventricular tachycardia originating from the left free ventricular wall sharp deflections were also recorded, ${ }^{811}$ thus suggesting the presence of extra tissue. Fascicular potentials, however, were present in three of our patients without clinical evidence of fascicular tachycardia and in 15 controls in the series of Wen et al. ${ }^{11}$ Regardless of their nature, such potentials seem to allow a better targeting of the tachycardia substrate thus avoiding unnecessary deliveries of energy into, and consequent scarring of, the ventricles.

\section{MODE OF ENERGY}

High energy DC shocks have been previously used for ablation of fascicular tachycardia. ${ }^{45}$ However, late malignant ventricular arrhythmias and sudden death are recognised complications of high energy DC ablation. ${ }^{20}$ Low energy DC offers non-arcing shocks and has been used successfully for ablation of the atrioventricular junction and accessory pathways. ${ }^{21}$ The reported complications compare favourably with those encountered in radiofrequency current series ${ }^{22}$ and it seems that both modes of energy probably offer safe treatment. The efficacy of radiofrequency current in this particular setting cannot be judged from our series. It would be interesting to see whether the small, localised lesion produced by radiofrequency is enough to eradicate the focus of this tachycardia.

\section{STUDY LIMITATIONS}

The main limitation of our study as well as of the other two series is the short follow up of the patients. In a recent report of 54 patients who underwent radiofrequency ablation of the AV junction, two patients with organic heart disease died suddenly. ${ }^{23}$ The long-term safety of ablation procedures targeting the ventricular myocardium is not yet established.

The recording of sharp deflections during tachycardia or sinus rhythm is a reliable and reproducible sign for the guidance of selection of ablation sites in patients with fascicular tachycardia. This approach should facilitate the procedure and ensure the avoidance of unnecessary energy discharges into the ventricle. The origin of these potentials seems to be the network of the posterior left fascicle but verification of their physiological significance has not yet been possible.

1 German LD, Packer DL, Bardy GH, Gallagher JJ. Ventricular tachycardia induced by atrial stimulation in patients without symptomatic cardiac disease. $A m, \mathcal{F}$ Cardiol 1983;52:95.

2 Ward DE, Nathan AW, Camm AJ. Fascicular tachycardia sensitive to calcium antagonists. Eur Heurt $\mathcal{f} 1984.5$ 896-905.

3 Ohe T, Shinomura K, Aihara N, Kakamura S, Matsuhisa $M$, Sato I, et al. Idiopathic sustained left ventricular $\mathrm{M}$, Sato I, et al. Idiopathic sustained left ventricular tachycardia: clinical and electron

4 Ruffy R, Kim SS, Lal R. Paroxysmal fascicular tachycardia: electrophysiologic characteristics and treatment by

5 Frank R, Tonet J, Rougier I, Gallais Y, Fontaine G, Grosgogeat Y. Endocardial fulguration for the treatment of ventricular tachycardia. Experience with 69 cases. Arch
Inst Cardiol Mex 1991;61:91-9.

6 Kim YH, Leitch JW, Klein GJ, Yee R, Teo WS, Lerther RA. Catheter ablation of idiopathic exercise-induced right bundle branch block and left axis deviation ventricular tachycardia. Can $\mathcal{f}$ Cardiol 1991;7:189-92. 
7 Page RL, Shenasa H, Evans JJ, Sorrentino RA, Wharton $M$, Prystowsky EN. Radiofrequency catheter ablation of idiopathic recurrent ventricular tachycardia with right idiopathic recurrent ventricular tachycardia with right
bundle branch block, left axis morphology. PACE bundle branch

8 DeLacey W, Nath S, Haines DE, Barber MJ, DiMarco J Adenosine and verapamil-sensitive ventricular tachycardia originating from the left ventricle: radiofrequency catheter ablation. PACE 1992;15:2240-4

9 Katritsis D, Ahsan A, Anderson M, Camm AJ, Ward DE, Rowland E. Catheter ablation for successful management of fascicular tachycardia. An approach guided by the recording of fascicular potentials [abstract]. $\mathcal{F} \mathrm{Am}$ Coll Cardiol 1994;36A.

10 Nakagawa $\mathrm{H}$, Beckman $\mathrm{KJ}$, McClelland JH, Wang $\mathrm{H}$, Arruda M, Santoro I, et al. Radiofrequency catheter ablation of idiopathic left ventricular tachycardia guided by a Purkinje potential. Circulation 1993;88: 2607-17.

11 Wen M-S, Yeh S-J, Wang C-C, Lin F-C, Chen I-C, Wu D. Radiofrequency ablation in idiopathic left ventricular tachycardia with no obvious structural heart disease. Circulation 1994;89:1690-6.

12 Ohe T, Aihara N, Kamakura S, Kurita T, Shimiuzu W, Shinomura K. Long-term outcome of verapamil sensitive sustained ventricular tachycardia in patients without apparent structural heart disease [abstract]. Circulation 1992;86:I-266.

13 Morady F, Kadish A, Rosenheck S, Calkins H, Kou WH, deBuitleir $M$, et al. Concealed entrainment as a guide for catheter ablation of ventricular tachycardia in patients
with prior myocardial infarction. $\mathscr{f} \mathrm{Am}$ Coll Cardiol with prior my

14 Miller JM, Harken AH, Hargrove WC, Josephson ME, Orishimo TF. Pattern of endocardial activation during sustained ventricular tachycardia. F Am Coll Cardio 1985;6:1280-7.

15 Fitzgerald DM, Friday KJ, Yeung Lai Wah JA, Bowman
AJ, Lazzara R. Myocardial regions of slow conduction participating in the reentrant circuit of multiple ventricular tachycardias: report on ten patients. $\mathcal{F}$ Cardiovasc Electrophysiol 1991;2:193-206.

16 Trappe Hysiol Klein $\mathrm{A}$ Auricchio A, Wenzlaff $\mathrm{P}$ Lichtlen PR. Catheter ablation of ventricular tachycardia: role of PR. Catheter ablation of ventricular tachycardia: role of the underlying etiology

17 Klein LS, Shih H-T, Hackett K, Zipes DP, Miles WM. Radiofrequency catheter ablation of ventricular tachycardia in patients without structural heart disease. Circulation 1992;85:1666-74.

18 Wilber DJ, Baerman J, Olshansky B, Kall J, Kopp D. Adenosine-sensitive ventricular tachycardia. Clinical characteristics and response to catheter ablation. Circulation 1993;87:126-34.

19 Coggins DL, Randall JL, Sweeney J, Chein WW, Van Hare G, Epstein L. Radiofrequency catheter ablation as a cure for idiopathic tachycardia of both and right ventricular origin. $\Im \mathrm{Am}$ Coll Cardiol 1994;23:1333-41

20 Evans GT, Scheinman MM, Bardy G, Borggrefe M, Brugada P, Fisher J. Predictors of in-hospital mortality after DC catheter ablation of atrioventricular junction: results of a prospective, international, multicentre study. Circulation 1991;84:1924-37.

21 Katritsis D, Heald S, Bashir Y, Ward D, Rowland E. Lowenergy ablation of accessory pathways for cases in which
radiofrequency ablation has failed. $\mathrm{Am} f \mathrm{Cardiol}$ 1993;72:1211-2.

22 Lemery R, Talajic M, Roy D, Coutu B, Lavoie L, Lavallee $\mathrm{E}$, et al. Success, safety and late electrophysiological outcome of low-energy direct-current ablation in patients with the Wolff-Parkinson-White syndrome. Circulation 1992;85:957-62.

23 Olgin JE, Scheinman MM. Comparison of high energy direct current and radiofrequency catheter ablation of the atrioventricular junction. $\mathcal{F}$ Am Coll Cardiol 1993;21: $557-64$. 